\title{
Probabilistic Methods based on Energy's Threshold in Switching Mode of Mobile Wireless Sensor for Surveillance Application
}

\author{
Amira Bendjeddou \\ LRS Laboratory \\ Department of Computer \\ Science \\ University of Annaba, Algeria
}

\author{
Nacira Ghoualmi \\ LRS Laboratory \\ Department of Computer \\ Science \\ University of Annaba, Algeria
}

\begin{abstract}
The continuity running of the sensor network on limited energy resources constitutes a real challenge for researchers. In fact, many schemes have been proposed to economize the wasted energy of this latter. However, most of them are proposed for static network. In this article we propose threshold formula and we present two probabilistic methods to reduce the energy consumption and at the same time improve the latency in mobile wireless sensor network surveillance applications, based ZebraNet. The first is PMFT for Probabilistic method with fixed threshold. The second is PMVT for probabilistic method with varying threshold. PMFT defines a fixed threshold for all nodes, which is calculated using Poisson distribution. Whereas, PMVT uses a variant threshold for each node with taking into account the mobility pattern and the residual energy of each sensor. The experimental evaluation shows that our methods outperform conventional methods, in terms of consumed energy and end to end delay in spite of the sensors mobility.
\end{abstract}

\section{General Terms}

Probabilistic methods, Maximize the network lifetime, an energy saving plan, threshold energy, switching mode, mobile wireless sensor.

\section{Keywords}

Surveillance, sleep/wakeup method, interferences, Poisson process, Marcov Chain, energy consumption, mobile sensor

\section{INTRODUCTION}

During the last decade, wireless sensor network (WSN) has attracted a lot of interests by its deployment in different fields such as military, medical and environment surveillance.

WSN consists of a large number of small, inexpensive and low power devices (called sensor nodes), scattered over a large field for sensing tasks. Typically, a sensor node includes four main units: sensing unit for data collection from the surrounding environment, processing unit for local data processing and storage, communicating unit which communicates data to a central collection point (sink node or base station) using multi-hop link, and the power unit that often consists of a battery with limited energy. In addition, this battery is irreplaceable because of the random deployment, or the hostility of the environment where it is placed in. On the other hand, WSN mustn't be interrupted and must be operated as long as possible to achieve the application requirements. Therefore, the crucial challenge of such networks is to ensure their survivability and prolong their network lifetime. So designing an energy saving plan is necessary to minimize the energy conception and to maximize the network lifetime. Obviously, many works have been proposed in this case. For instance, designing an efficient wakeup scheduling scheme [1], power control scheme [2], and designing aware routing protocol [3].

The majority of existing wireless sensor network applications require static network. However, recently applications that necessitate mobile sensors emerge. The Zebranet application [4] is an example of WSNs mobile that allows the study of zebra's behavior, and the SWIM application [5] that is used in the context of monitoring of whales. In this paper we propose two probabilistic methods for controlling the switching mode of the sensors in mobile wireless sensor network surveillance applications, specifically ZebraNet, they're Called PMFT (Probabilistic method with fixed threshold) and PMVT (probabilistic method with varying threshold). PMFT and PMVT aim at reducing the consumed energy and decreasing the end-to-end latency. The main ideas of these methods are: first, each sensor node periodically, in the wakeup period, checks its remaining battery. If this latter is less than a calculated threshold, the node will change its sate to relaying mode. Otherwise it remains on sensing mode. Second, when the node has data to send, the choice of relaying node is based on two metrics: the residual energy and the node interference in order to improve the end to end delay. The first method PMFT defines a fixed threshold for all nodes, which is calculated using Poisson distribution. Whereas, PMVT uses a variant threshold for each node, this latter is calculated based on two metrics: the mobility pattern and the residual energy.

The remainder of this paper is structured as follows. Section 2 presents the power conservation mechanism in WSN followed by the description of the probabilistic methods, simulation results and analysis of probabilistic methods in section 3, while section 4 concludes the paper.

\section{RELATED WORKS}

Several approaches have been proposed to minimize the consumed energy at MAC and routing layer. In fact, most of these works emphasize in stationary networks [6].

In [7] ATPC (Adaptive Transmission Power Control algorithm for wireless sensor networks) is proposed. The design was made based on empirical results from experiments done on three different sites (Grass Field, Parking Lot and Corridor). In [8] the authors propose ODTPC (On Demand Transmission Power Control algorithm), this algorithm allows each node to adjust dynamically its power transmission over time and to maintain a good link quality while reducing the initialization overhead in determining the optimal transmission power level. The authors of [9] propose a distributed power control algorithm for wireless sensor 
network. The design of this algorithm is based on experiences done in order to study the relationship between the link quality and the transmission power. In [10] an experimental approach is conducted to examine the benefit of power control in mobile wireless sensor network. In [11] the authors propose DPCS (A Distributed Power Control Scheme for Mobile Wireless Sensor Networks). This scheme aims at minimizing the consumed energy of each mobile node and at the same time it achieves a good connectivity of the network. Another category of schemes that aims at achieving power-efficient communication is the sleep/wakeup scheme. This type of scheme reduces radio energy that is wasted in idle state by turning off radio when it is not used.

In [12] the authors propose DW-MAC (A Low Latency, Energy Efficient Demand Wakeup MAC Protocol for Wireless Sensor Networks), which is the synchronized duty cycle MAC protocol, this scheme reduces end-to end delay in dense network by allowing nodes to wake up on demand during the sleep period of an operational cycle, in order to transmit or receive a packet. In [13] a sleep/wake scheduling scheme called SMED (Sleep/Wake Scheduling Scheme for Minimizing End-to- end Delay) is proposed to minimize the end-to-end delay for event driven multi-hop wireless sensor networks. The latter performs scheduling that is dependent on traffic load of each node.

\section{PROBABILISTIC METHODS}

\subsection{Probabilistic methods with Fixed threshold (PMFT)}

This method uses fixed threshold for all nodes which remains static over time. In this method all nodes periodically check their remaining battery, if the latter is less than a fixed threshold, node changes its mode from sensing mode to relaying mode. Otherwise node remains in sensing mode. Moreover, when node has data to be transmitted, it chooses one among their neighbors, node with maximum residual energy and minimum interference. PMFT consists of two main phases: state switching phase and relay choice phase which will be described below, but before we show how the threshold is calculated.

\subsubsection{Calculation of threshold}

The threshold represents the remaining amount of battery, which allows the node to continue its activity in the network. In network surveillance application, the events occur randomly, independently of one another and unpredictable over time. In this situation we say that our events follow Poisson process [14].

As our method is applied to zebraNet application we model the movement of zebras as an event.

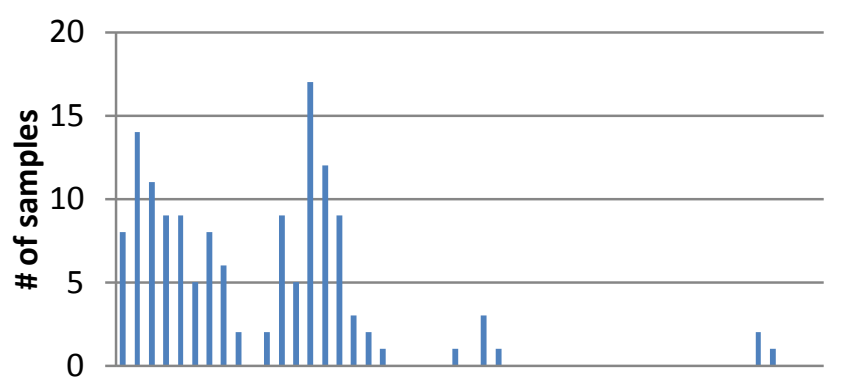

1471013161922252831343740434649

net distance traveled ( meters)

Fig 1: Distribution of zebra movements observed by the field biologists [4]

As shown in Figure 1[4], the Zebras (nodes) move dynamically and arbitrarily. So the movement of zebras is unpredictable over time. In this way, the threshold is calculated using the Poisson distribution as indicated below:

threshold $=E_{\text {Tot }} \times \alpha$

Where:

$\mathrm{E}_{\mathrm{Tot}}$ is the total consumed energy. This is calculated by using the general model described as follow:

$E_{T o t}=E_{t x}+E_{r x}$

$E_{T x}=T \times P_{T x}$

$E_{r x}=T \times P_{r x}$

Where:

$\mathrm{E}_{\mathrm{Tx}}$ : Energy spent at transmission,

$\mathrm{E}_{\mathrm{rx}}$ : Energy spent at reception,

$\mathrm{t}:$ is the necessary time for transmission,

$\mathrm{P}_{\mathrm{Tx}}$ : is the transmission power and

$\mathrm{P}_{\mathrm{rx}}$ : is the reception power.

$\alpha$ :is the probability that there are exactly $k$ occurrences $(k$ being a non-negative integer, $k=0,1,2, \ldots)$ in given interval of time $\lambda . \alpha$ is calculated using the following formula:

$\alpha=\frac{\lambda^{K} e^{-\lambda}}{K !}$

Where:

$e:$ is the base of the natural logarithm $(e=2.71828 \ldots)$.

$k:$ is the number of occurrences of an event.

$k !$ is the factorial of $k$

$\lambda:$ is a positive real number, equal to the expected number of occurrences during the given interval.

\subsubsection{The proposed method description}

Following the detailed description of PMFT phases

- State switching phase

In this phase each node periodically changes its state according to the threshold. 
1. First, all nodes are synchronized to sleep/ wakeup at the same time. And the threshold is calculated at the initial deployment of sensor nodes.

2. In the wakeup period, each node checks its remaining battery.

3. If the remaining battery is less than a calculated threshold, node changes its state to relaying mode.

4. Otherwise node remains on sensing mode.

5. The above steps (2 to 4 ) are repeated during the WSN lifetime.

\section{- Relay choice phase}

In This phase, sensor node with pending data chooses relaying node based on two metrics: The remaining energy and the interference rate; it will choose the relaying node with maximum remaining energy and minimum interference. Our choice of these metrics is argued on the following points:

First, Sensors are supported by a limited amount of energy. If a node exhausts its energy, so it will be considered as dead because it can not realize its tasks. In this way, the death of one node or more may cause a partial interruption or full of network communication. Hence, the network will be dead. Also the periodic selection of relaying node can balance the energy consumption over the network.

Second, if the topology has a large interference, this will generate collision and also the end to end delivery delay may be significant in the network. So reducing interference minimizes the packet retransmission which affects energy consumption and network lifetime.

This phase is shown as follow:

1. The interference of nodes and remaining energy are calculated periodically.

2. When node has data to be transmitted, it chooses one among their neighbors, the relaying node with maximum remaining energy and the minimum interference.

3. After that, it will send its data to the relaying node with maximum remaining energy and minimum interference.

In order to get node interference, we have used the interference model proposed in [15]. In this model, the total value of interference of node " $b$ " is given by following equation:

$I(b)=\sum_{a \in S_{b}} \frac{T_{a}}{d_{b a}^{k}}+\sum_{c \in\left(S_{2 R}-S_{b}\right)}\left(\frac{2}{3}\right) k \frac{T_{c}}{R^{k}}$

Where:

$\mathrm{S}_{\mathrm{b}}$ : is the set of all neighbor nodes in the communication range.

$\mathrm{S}_{2 \mathrm{R}}$ : is the set of all 2 hop neighbor nodes.

$\mathrm{T}_{\mathrm{a}}$ and $\mathrm{T}_{\mathrm{c}}$ : is the packet delivery rate of node.

$d_{b a}$ is the distance between node $b$ and $a$.

$\mathrm{R}$ : is the transmission range of node.

$\mathrm{K}$ is the index, $\mathrm{K} \epsilon[2,4]$.

Note that in our method we don't use the historical information to calculate the interference of node.

\subsubsection{Experimental evaluation}

In order to evaluate the overall performance of PMFT, we used simulations for measuring the consumed energy and the end to end delay. As it was mentioned earlier, our method is designed for mobile wireless sensor network surveillance application precisely zebraNet. For that, in the different simulations scenarios, we used the same configuration of ZebraNet application. The threshold is calculated using the specified data in [16].We compare our method with other conventional methods named as follow:

1) Synchronous sleep/wakeup scheme: which uses a synchronous sleep/wakeup where all nodes sleep and wakes up at the same time.

2) Greedy protocol [17]: In greedy protocol, node which has data to send, forwards packet to closest neighbor to the sink.

We implement these two methods and our method PMFT, with WSNet simulator [18] dedicated to sensor networks.

\subsubsection{Evaluation metrics}

- The Consumed energy

In our simulation, we are interested to the energy consumed at transmission and reception. In this context, we use the formula (2) described above.

\section{- The Average delivery delay}

The average delivery delay is defined as the average of the elapsed time between the transmission of packet and the reception of this packet at the sink.

$$
\text { Average delay }=T_{\text {rec }}-T_{\text {trans }} \text {. }
$$

Where:

$\mathrm{T}_{\text {rec }}$ : reception time of the packet at sink.

$\mathrm{T}_{\text {trans }}$ : transmission time of the packet.

The simulation setup and the different results will be described in the following sections.

\subsubsection{Simulation setup}

In our simulations, we use $20,40,60,80$ and 100 nodes. The sensor nodes are distributed randomly in the square area of $200 \times 200 \mathrm{~m}^{2}$. We use a single sink which is situated in the center of the area. The sensor nodes are randomly moving with a random speed, which vary in the interval of 0 to 18 $\mathrm{m} / \mathrm{s}$. The complete assumptions are summarized in Table 1 .

Table 1. The simulation parameters

\begin{tabular}{|l|l|}
\hline \multicolumn{1}{|c|}{ Parameters } & \multicolumn{1}{c|}{ Values } \\
\hline Area size & $200 \mathrm{~m} \times 200 \mathrm{~m}$ \\
\hline Simulation duration & $50 \mathrm{~s}$ \\
\hline Number of sensors & $20,40,60,80,100$ \\
\hline Max transmission power & $0 \mathrm{dbm}$ \\
\hline Min transmission power & $-30 \mathrm{dbm}$ \\
\hline Max transmission range & $200 \mathrm{~m}$ \\
\hline Initial battery capacity & $60 \mathrm{dbm}$ \\
\hline
\end{tabular}




\subsubsection{The results}

In this section, we evaluate the performance of our method in term of average consumption of energy and average end to end delay.

- The average consumption energy according to the time and network densities

Figure 2.a shows the average consumed energy over time for 20 sensors. We observe at each time that the consumed energy in PMFT is less than synchronous sleep/ wakeup scheme. This is explained by the fact that, when sensor node reaches the calculated threshold, it changes its mode from sensing to relaying mode. In this case, node consumes energy only to relay data so it can economize its energy. We show also in figure 2.b, that our method outperforms synchronous sleep/wakeup scheme with different network densities. This means that when nodes change their mode to relaying mode, they can decrease the consumed energy. Also the introduction of residual energy as criteria for choosing relay node plays an important role in the preservation of energy. So, periodically, when node has data to be transmitted, it will choose the relay node with a maximum residual energy, this periodic election decreases the wasted energy and at the same time balances the energy consumption in the entire network.

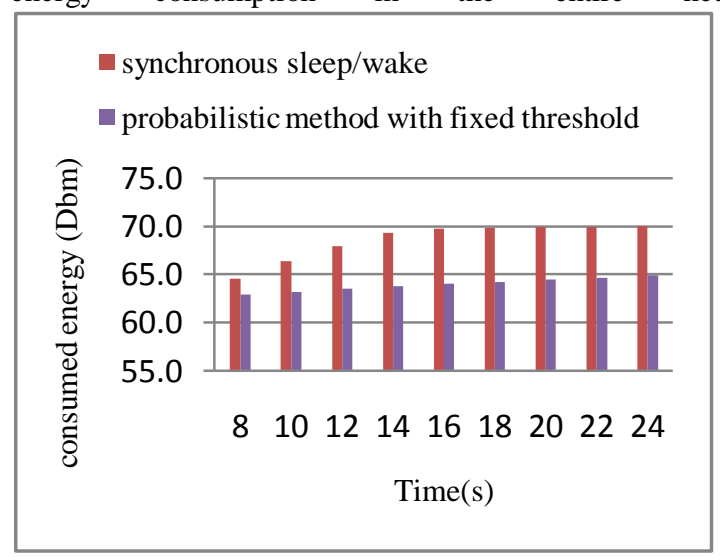

Fig 2.a: The average consumed energy over time

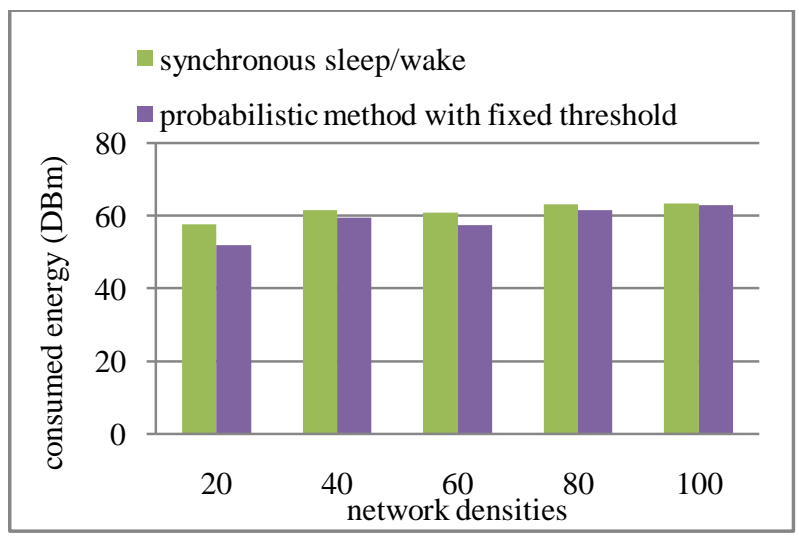

Fig 2.b:The average consumed energy with different network densities

\section{- The average delivery delay under different network} densities

Figure 3.a shows the average delay of PMFT and synchronous sleep/wakeup scheme under different network densities. In synchronous sleep/wakeup schemes, the Average delay increases with increasing of sensor's number contrary to our method. This is explained by when the number of node increases the traffic load of the network increases as well. And this will produce a higher overall interference, causing more collisions, that's why the end- to-end delay increases. We notice that PMFT reduces end-to-end latency. The reason lies in the introduction of interference as criteria for choosing relay node. So, minimum of collision can happened, in this case the end to end delay will be improved. In figure 3.b we show the average delay of the PMFT and greedy protocol. We see, when the density of the network increases, the average delay in greedy protocol increases too significantly contrary to PMFT. In greedy protocol the node chooses the closest node to the sink for relaying its data. Thus, the higher interferences are generated at the receiver. For this reason, a significant latency is caused. While in our method the node which has data to be transmitted, it chooses the relay node which has the minimum interference. This allows a significant reduction in collisions, which minimize the latency.

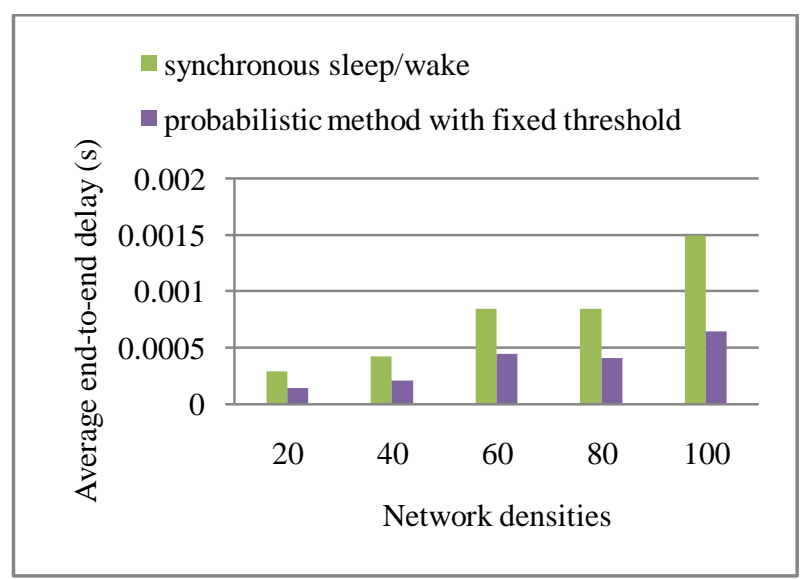

Fig 3.a:Comparaison of PMFT with synchronous sheme in term of average end-to-end delay

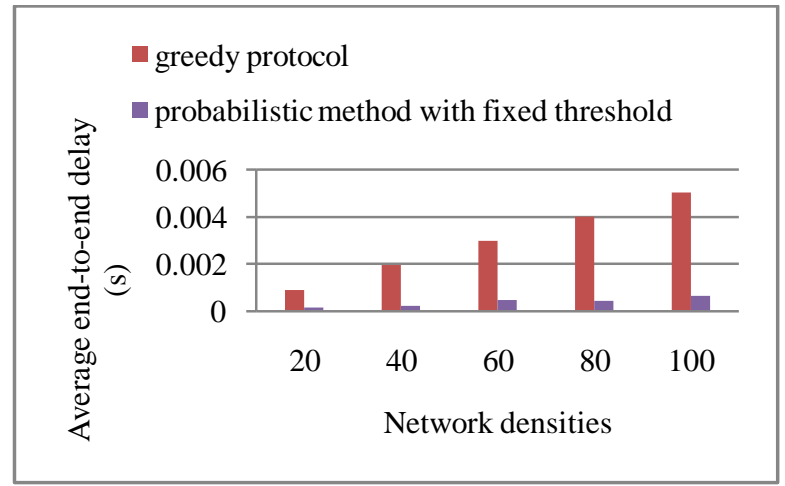

Fig 3.b : Comparaison of PMFT with greedy protocol term of average end-to-end delay . 


\subsection{Probabilistic method with varying threshold (PMVT)}

\subsubsection{Problematic}

In biological surveillance applications, specifically wildlife tracking, the animal's movement changes frequently and randomly as events occur. For instance, animals can change their speed in the presence of predator or for searching food. Thus when the speed of node increases, the consumed energy increases too. In the other hand, animals are collared with sensors which have different batteries capacities. For example smaller animals may need lower sensor in term of weight, these latter have small batteries, thus the residual energy of collars will be smaller. In the second variant of the probabilistic method we take these factors into account in order to minimize the wasted energy by each node.

\subsubsection{Biologist's model}

Biologists [4] show that zebra's movement can be described by three main states: grazing, graze walking, and fast moving (figure 4). Zebras spend most of their time grazing day and night. When zebras are in grazing state their movement rate is low.

At other times, zebras are in graze walking state, in this latter zebras move randomly, and they are faster than before. The movement is characterized by a higher speed than grazing state. Occasionally, zebras move more quickly, for long distance, either due to the presence of predator or because of the exhaustion area's vegetation. This movement is referred to fast moving state.

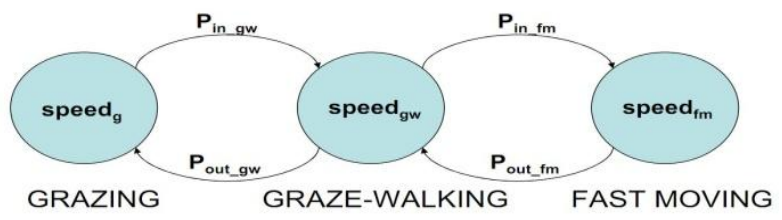

Fig 4: The three tired mobility model [4]

\subsubsection{Contribution}

Inspired from biological model, the second variant of the probabilistic method differs from the first in:

1. The mobility pattern of each node

2. The residual energy of each node.

3. Defining and using the threshold: The threshold is calculated using (1) and (2) metric.

4. The mobility pattern and the residual energy vary from one node to another.

\subsubsection{The proposed threshold Formula}

We propose to calculate the threshold for each node as follows:

Threshold $=\frac{E_{\text {tot }}}{E_{\text {res }}} * E_{\text {tot }}+P * E_{\text {mob }}$

Where

$\mathrm{E}_{\mathrm{Tot}}$ is the total consumed energy. This energy is calculated by using the formula (2)

$E_{\text {res: }}$ : The residual energy of the battery.
P: The transition probability from one state to another state (mobility transition patterns).

$E_{\text {mob }}$ : The energy consumed by the mobility: consumption of energy increases proportionally to the speed of the sensor.

\subsubsection{The model}

As discussed earlier, nodes can change their speeds randomly over time, and the transition probability between the current speed and the next one depends only on the current speed. In this way, nodes change their states (mobility pattern) over time with the transition probability noted $P$. To obtain these probabilities we model our system using Marcov Chain.

\section{- Marcov Chain Model}

A Marcov Chain [19] describes the evolution of a process over time. The process can take a finite number on states $S$ $=\left\{\mathrm{s}_{1}, \mathrm{~s}_{2}, \ldots, \mathrm{s}_{\mathrm{n}}\right\}$. The process moves from state $\mathrm{s}_{\mathrm{i}}$ to next state $\mathrm{s}_{\mathrm{j}}$ with probability $\mathrm{p}_{\mathrm{ij}}$. And the transition between the current state to the next one depends only on the current state not on the sequence of states that preceded it. The probabilities $p_{i j}$ are called transition probabilities. The probabilities verifies: $0=<\mathrm{p}_{\mathrm{ij}}<=1$ and $\sum_{j=0}^{n} p_{i j}=1$. The transition probabilities are represented using a matrix which is called, transition matrix.

So the set of all states and transition matrix characterizes completely the Markov chain.

\section{- The proposed model}

Assuming that each node is a zebra, we model the zebra's movement with Marcov Chain. In Fact, our work is devoted to the mobility of zebras. Based on biologist's observation shown in the previous section; we define three classes for zebra's mobility:

- Low mobility corresponds to grazing state,

- Medium mobility corresponds to graze-walking,

- High mobility concerns fast moving.

Thus, a set of state $S$ contains three states: $S=\{$ low mobility, medium mobility, high mobility\}.

Figure 4 shows the possible transitions between states observed by biologists. For our model we are interested in the mobility of zebra and how long they will remain in a given state. For that reason and in addition to the transitions between states described by the biologists, we add three transitions called the state itself :\{ low mobility, low mobility\}, \{medium mobility, medium mobility\}, \{high mobility, high mobility\}.The different transitions between states are shown in the following diagram:

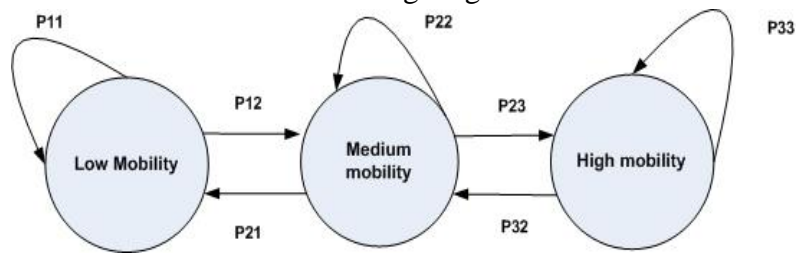

Fig 5: A diagram showing the transitions between states

Each transition between states occurs with probability P. we determine this probability, these probability are represented using transition matrix described below: 


$\mathrm{P}_{\mathrm{ij}=} \operatorname{\text {Low}}\left(\begin{array}{ccc}\text { Low } & \text { medium } & \text { High } \\ \mathrm{P}_{21} & \mathrm{P}_{22} & \mathrm{P}_{23} \\ & & \\ \mathrm{P}_{11} & \mathrm{P}_{12} & \mathrm{P}_{13} \\ \mathrm{P}_{31} & \mathrm{P}_{32} & \mathrm{P}_{33}\end{array}\right\}$

We determine the different transition probability ( $\mathrm{P}_{11}$, $\left.\mathrm{P}_{12}, \ldots\right)$ based on biologists experiments in [4], we obtain the following matrix:

$\mathrm{P}_{\mathrm{ij}}=\operatorname{\text {Medium}}\left\{\begin{array}{ccc}0.6 & 0.4 & 0 \\ 0.4 & 0.4 & 0.2 \\ 0 & 0.5 & 0.5\end{array}\right\}$

The first row in the matrix represents the different transitions probabilities of low mobility state to another state. Similarly the second and the third rows represent respectively, the probabilities of the transitions between medium mobility state and high mobility state to others

\subsubsection{The description of PMVT}

In this method we use a variant threshold for each node. At different time the node evaluates with formula (7) the appropriate threshold according to its mobility.

\section{- The State switching phase}

1. First, all nodes are synchronized to sleep/ wakeup at the same time.

2. In the wakeup period, each node calculates its own threshold based on its actual mobility using formula (7).

3. Each node checks its remaining battery:

If the remaining battery< threshold then

Node changes its state to relaying mode.

Else node remains on sensing mode.

4. The above steps 2 to 4 are repeated during the WSN lifetime.

\section{- The Relay choice phase}

This phase involves the same steps as described in PMFT.

\subsubsection{The experimental evaluation}

We evaluate the performance of PMFT through simulation experiments. We implement these methods by WSNet simulator. We are interested in, the average energy consumption and the end to end delay performance metrics. The simulation environment and the different simulation parameters will be described in the following sections.

\subsubsection{The Simulation setup}

We randomly place 20, 40, 60, 80 and 100 nodes, respectively in the square area of $200 \times 200 \mathrm{~m}^{2}$. We use a single sink which is situated in the center of the area. Nodes are mobile, they move randomly at a random speed generated in the interval of $[0,18] \mathrm{m} / \mathrm{s}$. The initial residual energy of nodes is set randomly to 40,50 and $60 \mathrm{dBm}$ each node has the ability to sense data from its surrounding environment then, it communicates it to the sink which is situated in the center of the area. Table 2 lists the complete assumptions used in our simulations.

Table 2. Simulation parameters

\begin{tabular}{|l|l|}
\hline Parameters & Values \\
\hline Area size & $200 \mathrm{mx} 200 \mathrm{~m}$ \\
\hline Simulation duration & $50 \mathrm{~s}$ \\
\hline Number of sensors & $20,40,60,80,100$ \\
\hline Max transmission power & $0 \mathrm{dbm}$ \\
\hline Min transmission power & $-30 \mathrm{dbm}$ \\
\hline Max transmission range & $200 \mathrm{~m}$ \\
\hline Max speed of sensors & $18 \mathrm{~m} / \mathrm{s}$ \\
\hline Initial battery capacity & $40,50,60 \mathrm{dbm}$ \\
\hline
\end{tabular}

\subsubsection{The results}

- The average consumption energy according to the time and network densities

Figure 6.a and 6.b compare PMVT with synchronous sleep/wake in term of the average consumed energy. More precisely, Figure 6.a shows the consumed energy for 20 nodes and figure 6.b shows the average consumed energy with different network densities. We observe that, each time and with different network densities PMVT consumes less energy than synchronous sleep/ wakeup scheme. This is explained by, based on its mobility, each node periodically checks its remain residual energy. If it is less than the calculated threshold, it changes its mode from sensing to relaying mode. Thus, node can decrease its wasted energy.

In the other hand, when a number of node increases, the traffic is important, so most of the energy is wasted for data transmission and reception. In such situation, when the node changes its mode to relaying mode, it wastes its energy only for relaying data. Therefore, our method outperform synchronous sleep/wake scheme in term of consumed energy with different network densities. 


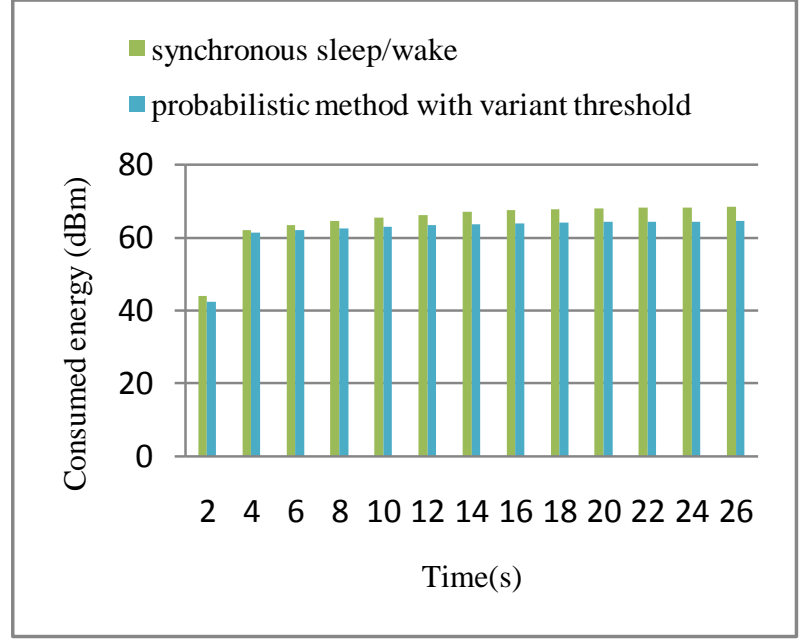

Fig 6.a: The average consumed energy over time

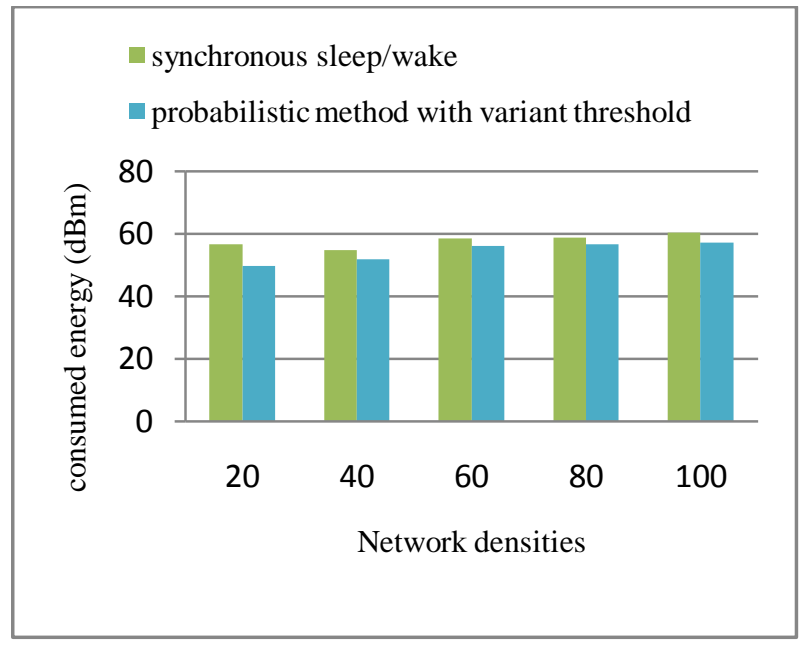

Fig 6.b: The average consumed energy over different network densities

\section{- The Average delivery delay under different network densities:}

Figure 7.a and figure 7.b compare our method PMVT with greedy protocol and synchronous sleep/wake scheme, respectively with different network densities. We observe that, in both synchronous sleep/wakeup scheme and greedy protocol the end-to-end delay increases with increasing of sensor's number. This is due to the heavy traffic generated by the network. Thus more collisions will happen. So the end- toend delay increases.

The above figures also illustrate that PMVT achieves lower end-to-end delay than other schemes. This is explained by the fact that in PMVT, we take into account. When the node has a packet to be transmitted, it chooses the relay node with minimum interference. In this case, the collision risk decreases, then the end-to-end delay decreases too.

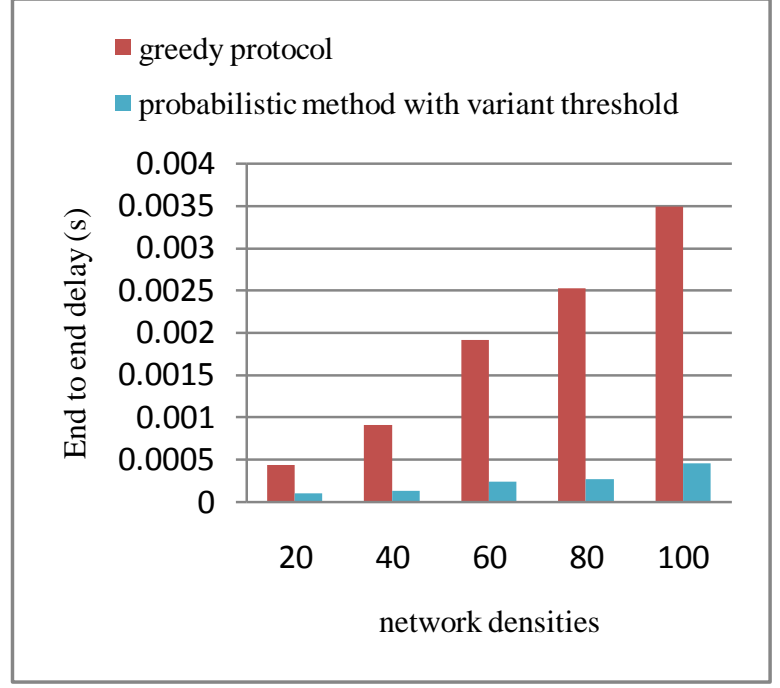

Fig7.a: A comparison of PMVT with synchronous sheme in term of average end-to-end delay

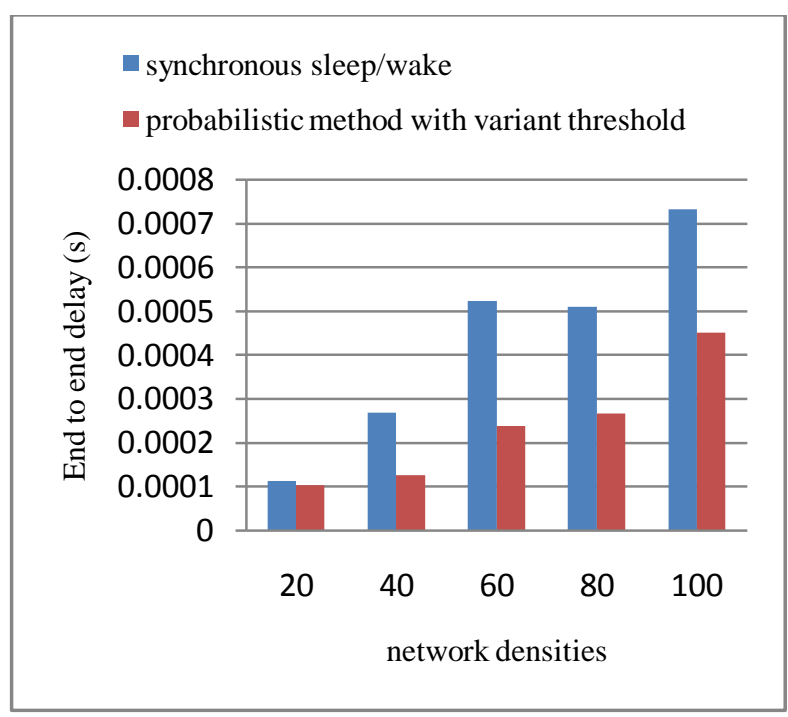

Fig 7.b:A comparison of PMVT with greedy protocol in term of average end-to-end delay

\section{CONCLUSION}

In this paper we have proposed two probabilistic methods for controlling the switching mode of the sensors in mobile wireless sensor network surveillance applications, specifically ZebraNet: PMFT (Probabilistic method with fixed threshold) and PMVT (probabilistic method with varying threshold). The first calculates the threshold by using poisson distribution. Whereas PMVT uses a variant threshold of each node which is calculated based on the mobility and residual energy of sensor. These thresholds are used to control the switching mode of sensors. When the remaining energy of sensor achieves a calculated threshold, sensor node changes its mode from sensing to relaying mode in order to minimize the wasted energy. Moreover these methods reduce the endto-end delay by introducing interferences as criteria for choosing relay node. The simulation results show that PMFT and PMVT outperform the conventional methods in term of consumed energy and end-to-end delay. 


\section{REFERENCES}

[1] A. Keshavarzian, H. Lee, L. Venkatraman. 2006. Wakeup scheduling in wireless sensor networks. In Proceedings of ACM MobiHoc, 322-333, Florence Italy.

[2] J. Wu, S. Yang. 2006. Optimal movement-assisted sensor deployment and its extensions in wireless sensor networks. In Proceedings of the International Conference on Parallel and Distributed Systems (ICPADS'06), 1215.

[3] Ruay-Shiung, C. and K. Chia-Jou. 2006. An energy efficient routing mechanism for wireless sensor networks. In Proceedings of 20th International Conference on Advanced Information Networking and Applications, IEEE Computer Society, Washington-DC, USA, $18-20$.

[4] P. Juang, H. Oki, Y. Wang, M. Martonosi, L. Peh, D. Rubenstein.2002. Energy-efficient computing for wildlife tracking: design tradeoffs and early experiences with Zebranet. In Proceedings of Architectural Support for Programming Languages and Operating Systems (ASPLOS).

[5] Z.J. Haas, T. Small.2006. A new networking model for biological applications of ad hoc sensor networks, IEEE/ACM Transactions on Networking, Vol. 14, $\mathrm{n}^{\circ} 1$, $27-40$.

[6] Gregory Hackman, Otav Chipara, and Chenyang Lu. 2008 Robust Topology Control for Wireless Sensor Networks. In Proceedings of the ACM Sensys, November 2008.

[7] S. Lin, J. Zhang, L. Gu, T. He, and J. Stankovic. 2006. ATPC: Adaptive Transmission Power Control for Wireless Sensor Networks. In Proceedings of SenSys'06, Nov 2006.

[8] Kim, J., S. Chang and Y. Kwon. 2008. ODTPC: On demand transmission power control for wireless sensor networks. In Proceedings of International Conference on Information Networking, 1-5.

[9] Jang- Ping Shen, Kun-Ying Hsieh and Yao-Kun Cheng. 2009. Distributed Transmission Power Control Algorithm for Wireless Sensor Networks. JOURNAL OF INFORMATION SCIENCE AND ENGINEERING, 1447-1463.
[10] JeongGil Ko, Andreas Terzis. 2010. Power Control for Mobile Sensor Networks: An Experimental Approach. In Proceedings of IEEE Communications Society Conference on Sensor, Mesh and Ad Hoc Communication and Networks.

[11] A. Bendjeddou, F. Djemili Tolba, N. Ghoualmi and P. Lorenz. 2011. DPCS: A Distributed Power Control Scheme for Mobile Wireless Sensor Networks. In proceedings of The Fourth IEEE International Symposium on Innovation in Information \& Communication Technology, Amman, Jordan, ISBN :978-1-61284-672-9, 30 - 34.

[12] Yanjun Sun, Shu Du, Omer Gurewitz, David B. Johnson. 2008. DW-MAC: A Low Latency, Energy Efficient Demand-Wakeup MAC Protocol for Wireless Sensor Networks. In Proceedings of. 9th ACM international symposium on Mobile ad hoc networking and computing.

[13] Babar Nazir, Halabi Hasbullah1 and Sajjad A Madani.2011. Sleep/wake scheduling scheme for minimizing end-to-end delay in multi-hop wireless sensor networks, EURASIP Journal on Wireless Communications and Networking, 1-14.

[14] Catherine Forbes, Merran Evans, Nicholas Hastings, Brian Peacock.2010. Statistical Distributions, ISBN-13: 978-0470390634, Edition 4.

[15] X.M. Zhang, Q. Liu, S.F. Dai, and Y.Z. Liu. 2009 Traffic Load-Based Interference-Aware Routing Protocol for Mobile Ad Hoc Networks, (in Chinese with English abstract) . Journal of Software, vol. 20, 2721-2728

[16] Ilya R. Fischhoff, Siva R. Sundaresan, Justine Cordingley, D. I. Rubenstein.2007. Habitat use and movements of plains zebra (Equus burchelli) in response to predation danger from lions, Behavioral Ecology, 1-5.

[17] B. Karp and H. T. Hung. 2000. GPSR: Greedy Perimeter Stateless Routing for wireless sensor networks. In Proceedings of 6th ACM MOBICOM, 243-254.

[18] Elyes Ben Hamida. 2009. Stochastic modeling and simulation of multi-hop wireless networks, $\mathrm{PhD}$ thesis, National Institute of Applied Science, lyon.

[19] Pierre Bremaud. 2008. Markov Chains, ISBN-13: 9780387985091, Springer; Corrected edition, March 1. 